\title{
Intervenciones
}

\section{para la reutilización \\ de la ex aduana marítima de Frontera, Tabasco, México}

Interventions for Reusing the Former Coast Customs Service Building at Frontera, Tabasco, Mexico

\section{Geiser Gerardo Martín Medina}

Universidad Autónoma de Yucatán (UADY), México

arqlgo_geisermartinmedina@hotmail.com

\section{Luis Fernando Guerrero Baca}

Universidad Autónoma Metropolitana (UAM), México

luisfg1960@yahoo.es

\section{Resumen}

Este INFORME expone los procesos de intervención que se desarrollan en uno de los monumentos más importantes del patrimonio edificado de Tabasco, México: la decimonónica ex aduana marítima de Frontera (puerto en el sureste del Golfo de México), la cual es notable por su historia, singularidad constructiva y calidad compositiva. Las obras en proceso buscan usar el potencial espacial del inmueble para acondicionarlo como museo dedicado a la navegación y, de este modo, incidir en la recuperación de la memoria histórica de una parte de dicha población que si bien aun hasta hace medio siglo tuvo gran relevancia hoy se ve absorbida por el desarrollo industrial. La intervención destaca por razón de que ha permitido rescatar procedimientos constructivos olvidados, como el manejo de bloques de conchuela, tejas marsellesas, y los morteros de cal y puzolanas.

\section{Palabras clave}

trópico húmedo; cal; puzolanas; bloques de conchuela; Aduana; Tabasco; México

\section{Abstract}

This REPORT describes the intervention processes that are taking place in one of the most important monuments of built heritage in Tabasco state, Mexico: the Nineteenth-century former Coast Customs Service Building at Frontera (a harbour in the southeast of the Gulf of Mexico), which is relevant for its history, fabric singularity and composition quality. Work in progress seeks to enhance the space potential of this construction for adaptation as a navigation museum and, thus, to use it as a vehicle to recover the historical memory of a town that until half a century ago had great significance, but which is now being absorbed by industrial development. The intervention stands out because it has allowed rescuing diverse forgotten construction procedures, such as the use of seashells blocks, marseille tiles, lime mortar and pozzolanic materials.

\section{Key words}

tropics; lime; pozzolanic materials; seashells blocks; Customs Service Building; Tabasco; Mexico 


\section{Introducción}

E I puerto de Frontera, en la actual cabecera municipal de Centla, Tabasco, México, se ubica en la margen oriental de la desembocadura del río Grijalva, a $18^{\circ} 31^{\prime}$ de latitud norte y $92^{\circ} 39^{\prime}$ de longitud oeste. El clima local es cálido húmedo, con lluvias casi todo el año, pero de mayor intensidad en verano. El régimen pluvial alcanza los 1695 mm anuales y las temperaturas van de los $20.5^{\circ} \mathrm{C}$ a $\operatorname{los} 30.8^{\circ} \mathrm{C}$, aunque la sensación térmica, causada por la elevada humedad relativa prevaleciente, es mucho mayor. En el tramo costero coexisten condiciones de palmares, selva alta y bosque tropical (Barrera 1989:81, 85).

Éste al que nos referimos es uno de los dos puertos más importantes de Tabasco, y está entre los 23 más destacados del país, al contar con tráfico marítimo constante derivado de actividades pesqueras y comerciales de alcance internacional, además de ser base de abastecimiento de plataformas marinas de Petróleos Mexicanos (Pemex); sin embargo, la escasa profundidad de su dragado no permite la llegada de grandes embarcaciones (Digaohm-Semar s. f.:1).

Existen múltiples evidencias de que ya en el periodo Clásico Temprano habitaban la región grupos mayas chontales, asentados en caseríos desde los que controlaban las rutas marítimas y fluviales de la desembocadura (Chávez 2007:133). A partir de 1519, los españoles se establecieron en la ribera opuesta del río, en un sitio en el que, si bien no se ha podido precisar, situaron la desaparecida Villa de Santa María de la Victoria, primera ciudad española que se fundó en el actual territorio mexicano, emplazada sobre los restos del asentamiento maya de Potonchán que Cortés destruyó durante la Batalla de Centla; los conquistadores, no obstante, se vieron obligados a abandonarla en el mismo siglo XVI, a consecuencia tanto de las difíciles condiciones del entorno geográfico como de la constante amenaza de los ataques piratas (Díaz 2007:54, 55, 273, 274).
Pasaron casi tres siglos para que el puerto se implantara definitivamente en su localización actual, lo cual ocurrió en 1780 gracias a la tenacidad del presbítero Tomás de Helguera (Ruiz en Barrera 1989:111). El poblado, de cualquier manera, no prosperó sino hasta 1817, cuando el militar español y gobernador de Tabasco, Francisco de Heredia y Vergara impulsó la ejecución de obras defensivas y urbanas para conformar el puerto de San Fernando de la Victoria, Ilamado así en alusión al rey Fernando VII de España y como recuerdo de la desaparecida Villa de Santa María (Ruiz en Barrera 1989:111).

El puerto jugó un papel trascendente al final de la guerra de Independencia, con lo que se consolidó de manera definitiva, y en 1826 se le denominó Villa y Puerto de Guadalupe de la Frontera, tanto para borrar la memoria de la monarquía española como para honrar al presidente Guadalupe Victoria (Barrera 1989:112). En 1928 se le designó Ciudad y Puerto Álvaro Obregón, pero en julio de 1947 recibió el sencillo nombre de Frontera (García 1996). No obstante que desde 1829 se estableció ahí una autoridad aduanera marítima, el edificio que la albergaría, obra ordenada por el entonces presidente de la República Benito Juárez (Barrera 1989:12), y no se concluyó sino hasta 1872 .

Se trata de un inmueble sobrio, de corte, evidentemente, neoclásico (Figura 1a), construido sobre un banco de arena que le ha dado notable estabilidad estructural. Tiene planta rectangular, con un desarroIlo perpendicular al río y dimensiones aproximadas de $42.75 \mathrm{~m}$ en el sentido oriente-poniente y casi 30 $\mathrm{m}$ en su eje norte-sur (Figura 1b). En cuanto a sus fachadas, la principal corresponde al paño poniente y su frente da hacia el río Grijalva, con el que se vincula mediante un amplio muelle y un área actualmente ajardinada, cuyo emplazamiento y forma sugiere que históricamente funcionó como explanada de descarga; las otras tres son sumamente austeras: la norte da a un angosto callejón de servicio que llega al río; el frente sur mira hacia una colindancia a través de un espacio también ajardinado, y, finalmente, la fachada oriente comunica el edificio con el poblado, en una ubicación estratégica que ayuda a conformar el perfil urbano en torno de la plaza central, casi delante de la parroquia principal y de los edificios administrativos y comerciales más destacados. A pesar de esta localización, la imagen de esta fachada, puesto que correspondía con el área de almacenamiento de la antigua aduana, es muy sencilla y, por lo tanto, contrasta con el cuidadoso diseño de la opuesta, frente al río, que pertenecía a la zona de oficinas.

Podría decirse que el edificio en planta se divide en tres núcleos: la zona abierta al poniente, junto al muelle, y dos sectores, el de oficinas, desarrollado en dos niveles, con la fachada principal hacia el río, y el de almacenamiento y servicio, al oriente, de un solo nivel, con un patio interior en su eje central y fachada que da a la plaza central (Figura 1b).

La estructura de mampostería del inmueble está hecha a base de singulares bloques de un sedimento marino calizo fuertemente consolidado al que regionalmente se denomina conchuela (Figura 2); aquéllos se cortaban y tallaban para darles perfiles paralelepípedos y conformar los muros de carga, dovelas de vanos y arquerías, así como las columnas de la zona de almacenamiento, que originalmente sostenían una sólida estructura de vigas y armaduras de madera, techada con teja de barro tipo marsellesa (Figura 2).

El núcleo de dos pisos cuenta con un corredor porticado que da al río, con imponentes arquerías soportadas por robustas pilastras de sección cuadrangular en la planta baja y columnas de orden toscano de sección hexagonal en la alta, lo que en conjunto define la fachada principal del edificio.

Los espacios interiores de este sitio de oficinas tienen gruesos muros en ambas plantas, los cuales sirven 

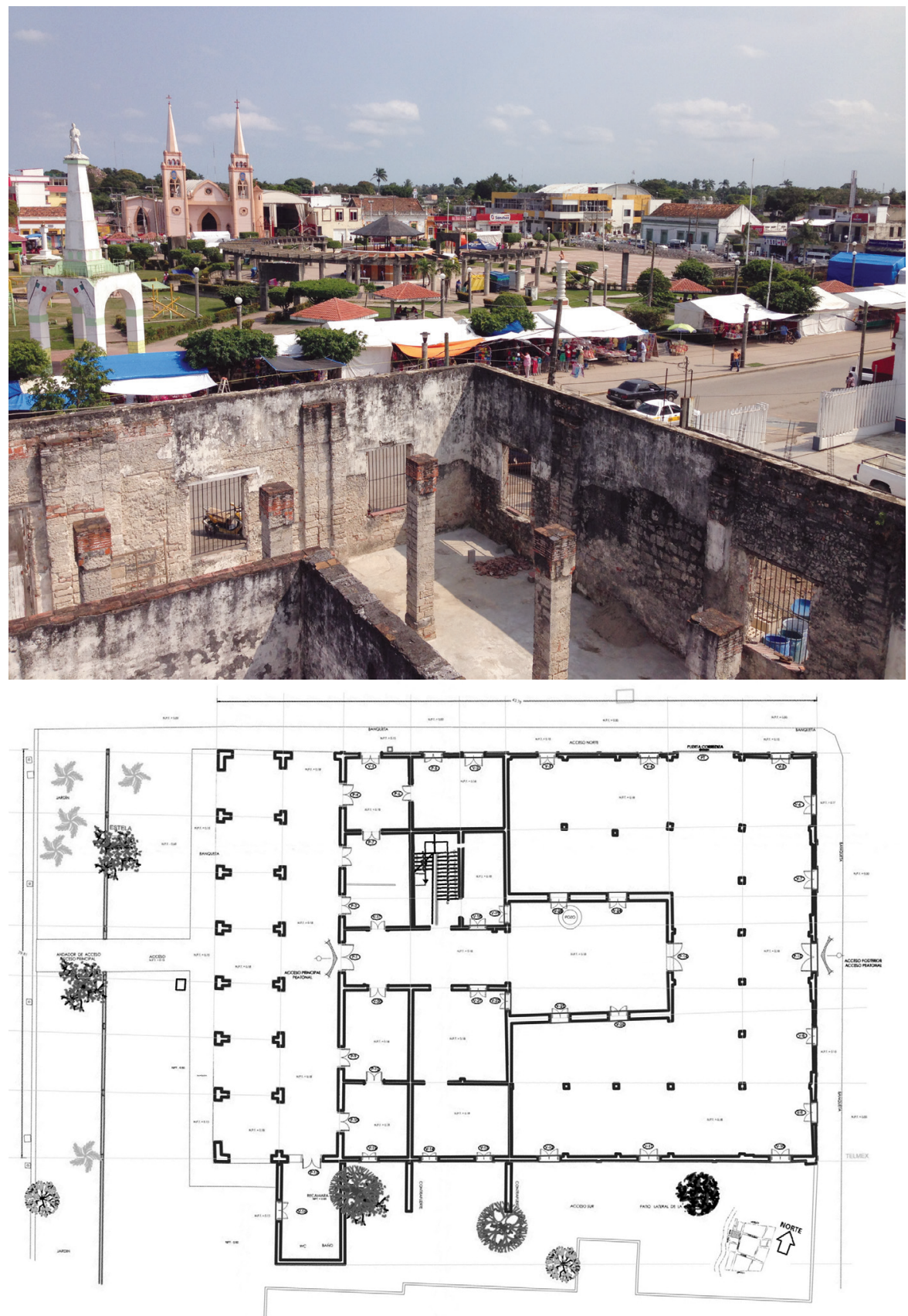

FIGURA 1. a) Área de almacenamiento de la ex aduana que da frente a la plaza central del puerto de Frontera (Fotografía: Luis Fernando Guerrero, 2015); b) Planta de conjunto del edificio antes de la intervención (Dibujo: Luis Fernando Guerrero, con base en los planos de la empresa constructora: Fernando Jiménez González).

FIGURA 2. Los bloques de conchuela de muros y platabandas quedaron expuestos a la intemperie al perderse paulatinamente los recubrimientos originales de cal y arena (Fotografía: Luis Fernando Guerrero, 2015).

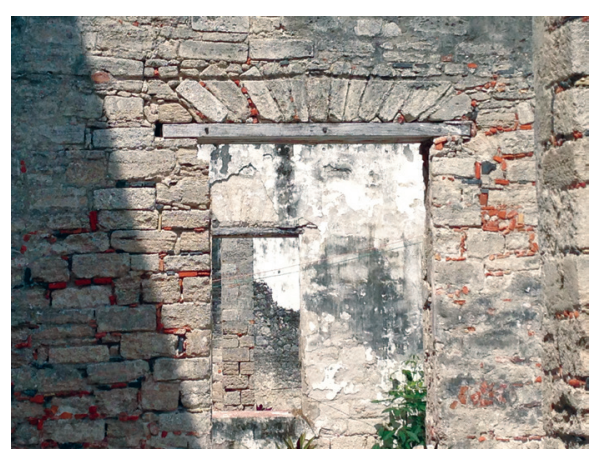

de apoyo a entrepisos y cubiertas configurados por bóvedas catalanas de ladrillo, cargadas por perfiles de acero.

Llama la atención que en imágenes antiguas se observa que la cubierta de esta parte del edificio era inclinada, a cuatro aguas, con tejas marsellesas, seguramente apoyadas en listones, largueros y armaduras de madera. Desafortunadamente, no se ha podido documentar en qué momento se perdió este sistema, que se transformó en un techo plano con azotea; la zona de almacenamiento en torno del patio central, en cambio, conservó las cubiertas originales de madera y teja, soportadas por muros y columnas aisladas, como se ve en fotografías tomadas durante los años setenta del siglo pasado (Figura 3).

\section{Los antecedentes a la intervención}

Esta imponente estructura, que estuvo destinada a funciones aduanales que daban servicio al sureste del Golfo de México y concluyeron a finales del siglo XX, cuando la actividad marítima comercial de la región decayó drásticamente, se convirtió en una sucursal bancaria (Barrera 1989:152). Sin embargo, las dificultades que implicaba su mantenimiento hicieron que el edificio se abandonara paulatinamente, hasta que la antigua zona de almacenamiento perdió su estructura de madera y los techos de teja colapsaron, con lo que más de la mitad de su espacio quedó como un enorme patio que terminó en depósito de autos custodiado por el municipio (Barrera 1989:156).

Gracias al interés y las presiones de la comunidad local, el inmueble se cedió al Instituto Estatal de Cultura (IEC, Tabasco, México), que lo convirtió en un espacio cultural: se propuso establecer un museo dedicado a la navegación debido a la importancia que esta actividad tuvo para el desarrollo del puerto (Ortiz 2015). Sin embargo, como esta institución carece de infraestructura, personal y 


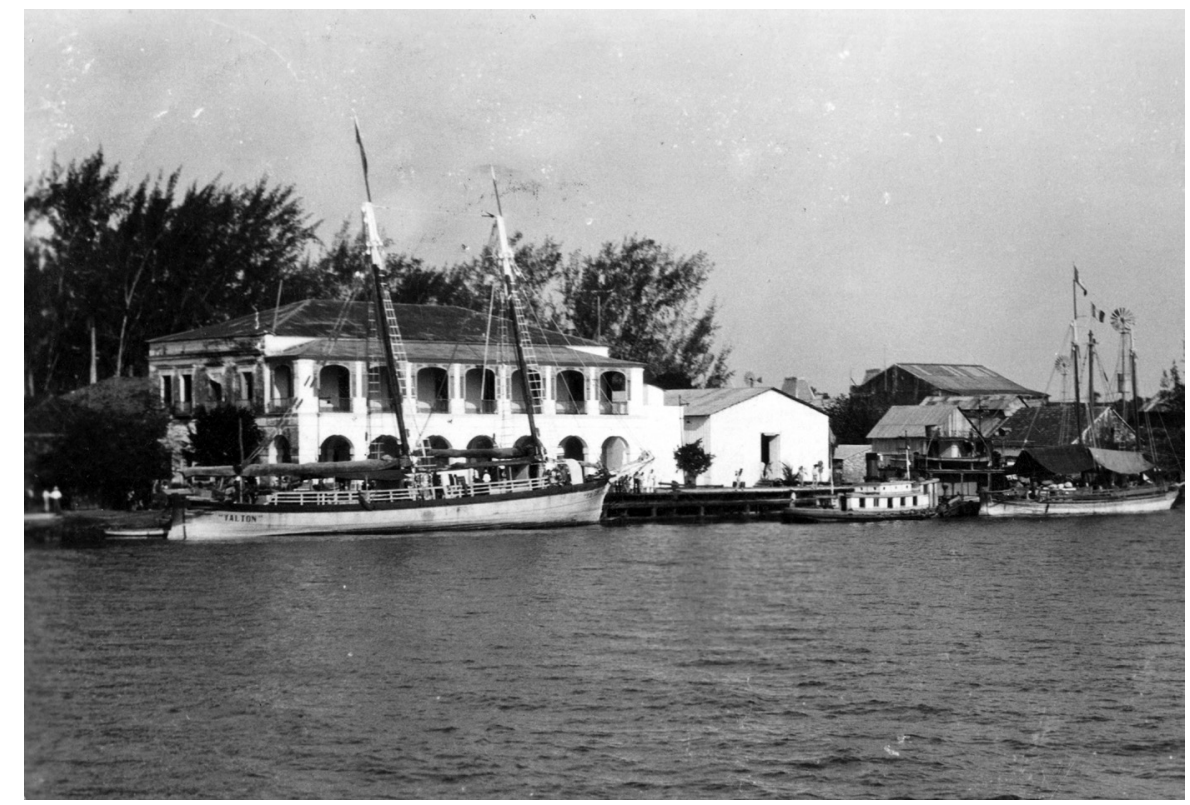

FIGURA 3. Detalle de una postal de 1940 del puerto de Frontera (Fuente: México en Fotos [www. mexicoenfotos.com], 2015).

equipamiento para ejecutar restauraciones, las obras necesarias para la intervención patrimonial se sometieron a licitación con el apoyo de la Secretaría de Ordenamiento Territorial y Obras Públicas (SOTOP) del estado.

Por las dimensiones del inmueble y los gastos que exigía su restauración, se planteó desarrollarla en varias etapas. La primera (2010) consistió en limpieza del conjunto y consolidación de la estructura. En la siguiente (2012) se intervino gran parte de los aplanados y se impermeabilizó la cubierta, con lo que el edificio se mantuvo en relativo equilibrio. Relativo, ciertamente, a la falta de techumbre en la zona de almacenamiento - aunada a la ausencia de puertas y ventanas-, el viento y la Iluvia afectaron nuevamente los elementos constructivos y se produjeron deterioros derivados del crecimiento de vegetación nociva que, en las condiciones climáticas imperantes, proliferó de forma vertiginosa (Figura 2).

En enero del 2015 se volvió a aprobar una licitación para la ejecución de obras de infraestructura en el puerto destinadas al desarrollo de la tercera etapa de restauración y habilitación de la ex aduana marítima de Frontera, Tabasco, cuyo proyecto, después que la delegación estatal del

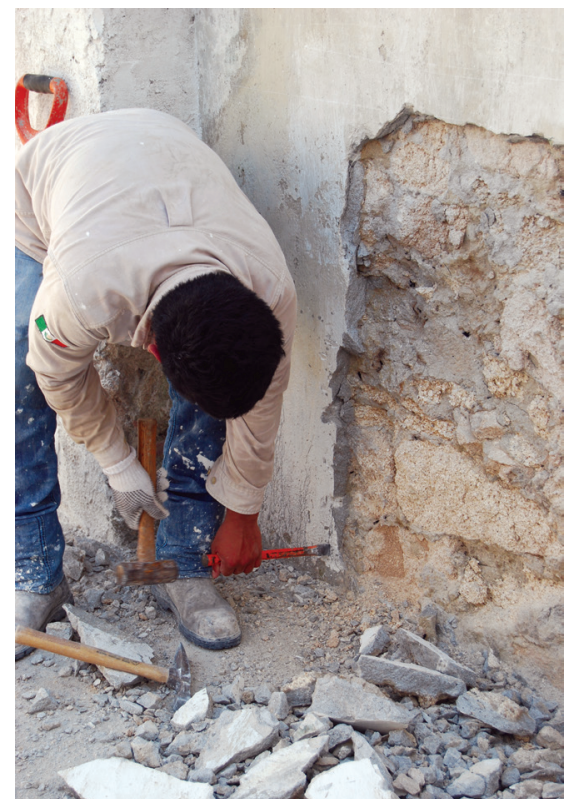

FIGURA 4. Eliminación de gruesas capas de aplanado de cemento que propiciaron que la humedad encapsulada disgregara algunos componentes del núcleo de los muros (Fotografía: Luis Fernando Guerrero, 2015).

Historia (INAH, México) lo revisó y aprobó, se sometió a concurso. No obstante que la empresa ganadora, cuya razón social es Fernando Jiménez González, cuenta con muchos años de experiencia en el desarrollo de proyectos y obras de gran escala, se acotó, debido a la singularidad del edificio, que su proyecto debía prever la participación de asesores en las áreas de arqueología y restauración de bienes inmuebles.

Durante las visitas previas al inicio de las labores, se observaron diversos tipos de deterioros; algunos derivados de las citadas afectaciones del medio ambiente, y la mayoría, producto, al parecer, de mezclas inapropiadas de sistemas constructivos, intervenciones inconclusas y la introducción inadecuada de instalaciones (Figura 4).

La afectación más compleja se causó por el uso de componentes de concreto armado y aplanados de cemento incorporados, probablemente, a mediados del siglo Xx. Aunque algunas secciones de estos elementos de concreto agregados se removieron durante las dos primeras etapas de restauración, y se integraron nuevamente aplanados a base cal y arena, en otras zonas esos nuevos revoques se aplicaron, lamentablemente, sobre superficies de cemento, con lo que se crearon daños colaterales derivados de la acumulación de humedad.

Por otro lado, durante ambas etapas de intervención las especificaciones para los recubrimientos se sustentaron en un criterio que comúnmente se propone en el centro y norte de México: incorporar mucílago de nopal a las mezclas con el fin de hacerlas más trabajables y ayudar al endurecimiento gradual de la cal. Sin embargo, la presencia de esta sustancia en un ambiente de excesiva humedad, como el que caracteriza a la región, sumada al hecho de que el inmueble se encuentra a la vera del río Grijalva, impidió un adecuado fraguado de los aplanados y propició una baja resistencia a la abrasión.

La delgada capa final de los aplanados se pulió con mezclas que contenían, además del mucílago, una pequeña proporción de cemento blanco, para "ayudar al fraguado", dar "resistencia" al intemperismo y, así, revertir los problemas de las intervenciones realizadas en 2010 y 2012. Lógicamente, las consecuencias no 
se hicieron esperar $y$, a tres años de haberse realizado aquellas obras, eran evidentes la presencia de humedad interna en los muros, el desarrollo de fisuras, el desprendimiento en bordes y el crecimiento de líquenes y microflora, aparte de que los revoques no tenían adherencia en los muros y se disgregaban con una leve presión de los dedos.

Otro problema de aquellas intervenciones fue consecuencia de su ejecución, ya que en las anteriores se buscó regularizar y "plomear" los muros para corregir las imperfecciones características de su proceso constructivo histórico, y, al engrosar algunas secciones de los aplanados, se causaron desprendimientos por su propio peso (Figura 5).

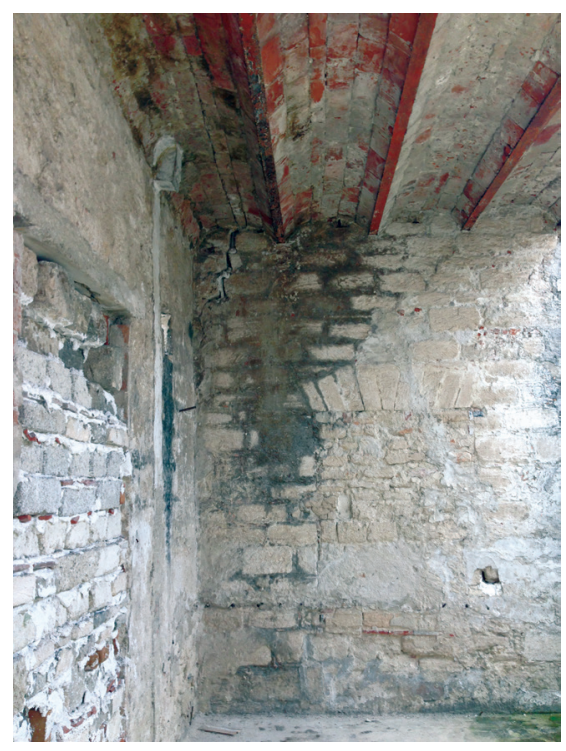

FIGURA 5. Los paños sin enlucir que presentaban irregularidades derivadas de la materia prima y la calidad de la mano de obra original (Fotografía: Luis Fernando Guerrero, 2015).

Pero la complicación más grave previa al inicio de las obras consistió en que, no obstante que las instancias pertinentes ya mencionadas habían revisado y aprobado el proyecto para su ejecución, contenía notables errores y contradicciones que, de haberse llevado a cabo, hubieran afectado de manera irreversible el inmueble patrimonial. Además de que las especificaciones seguían indicando la necesidad de usar el mucílago de nopal y de consolidar la estructura con mezclas de cal "enriquecidas" con cemento, se proponía una impactante modificación del sistema de cubiertas de la zona del patio, así como la incorporación de apoyos verticales de concreto armado, sostenidos por zapatas corridas perimetrales, adosados a las columnas históricas y a su cimentación de mampostería de bloques de conchuela.

Otras de las propuestas aprobadas - por mencionar sólo algunas- consistían en la demolición indiscriminada de espacios correspondientes a etapas posteriores a la edificación de la aduana, así como en la introducción de canalizaciones eléctricas y de drenaje pluvial a través de espacios clave del monumento. Se proponían, una para la planta baja y otra para la alta, sendas zonas de baños, lo cual preveía, además de adosar los muebles sanitarios a los muros históricos, realizar múltiples perforaciones por pisos, entrepisos y cimentación que hubieran generado, indudablemente, graves problemas de estabilidad al edificio, además de potenciales focos de humedad.

Tras varias visitas previas al desarrollo del nuevo proyecto, las instituciones involucradas en la ejecución de la obra aprobaron, con el apoyo de los asesores, la modificación radical de la propuesta.

\section{La obra de restauración ${ }^{1}$}

A fines del 2014, una vez que se replanteó y aprobó nuevamente el proyecto, y se adecuaron algunas especificaciones relacionadas con el manejo de la cal, las obras dieron inicio formal con labores de limpieza y habilitación de zonas de trabajo y almacenamiento. Se retiraron la vegetación menor de las zonas abiertas y el escombro perteneciente al inmueble, separando los elementos constructivos que pudieran reciclarse en labores futuras: pedacería de teja, ladrillo, mosaicos y bloques de conchuela.

\footnotetext{
${ }^{1}$ La información de este apartado proviene de Jiménez 2014 y el informe respectivo, en elaboración.
}

En el espacio abierto frente a la fachada poniente se realizó, de igual manera, la limpieza de vegetación y basura acumulada, en este caso, para poner un relleno del escombro acumulado y elevar el nivel original, lo que serviría para colocar dos artesas, de $4 \mathrm{~m} \times 3 \mathrm{~m}$ de base y 1.20 $\mathrm{m}$ de altura, para el apagado de cal. Ambas cuentan con una capacidad de saturación de 300 sacos de $25 \mathrm{~kg}$. Se cuidó especialmente el uso de cal química de $95 \%$ de pureza, que se dejó hidratar por periodos mínimos de 20 días. Con el fin de alcanzar un mejor fraguado de los morteros, dadas las condiciones de humedad del sitio, y, al mismo tiempo, dotarlos de la firmeza necesaria para su trabajo estructural, se decidió agregarles polvo de ladrillo y de tejas del escombro en una proporción de 1 a 4 respecto de la cal y generar, de este modo, reacciones puzolánicas. ${ }^{2}$ La dosificación de la arena y su granulometría obedecían, lógicamente, a la función que habrían de desarroIlar utilizando cuatro volúmenes de arena gruesa, uno de cal, un cuarto de polvo de ladrillo en recalces y reposiciones estructurales. Los acabados base tenían tres volúmenes de arena gruesa por uno de cal; los intermedios, dos de arena semifina por uno de cal, y los enlucidos finales, al igual que las lechadas para inyección de grietas, un volumen de arena fina, uno de cal y un cuarto de polvo de ladrillo (Figuras 6 y 7 ).

Paralelamente, se hicieron los estudios detallados de deterioros, que se registraron mediante fotografías y cédulas por cada sección del edificio. Se observó gran cantidad de mi-

\footnotetext{
${ }^{2}$ El hidróxido de calcio combinado con materiales de origen volcánico, o con arcillas calcinadas, genera silicatos cálcicos hidratados que les confieren a los morteros mayor resistencia mecánica y velocidad de fraguado incluso en condiciones de alta humedad. Estas reacciones se denominan puzolánicas, como referencia al tipo de arenas que se utilizaban en la época romana y que provenían de la región de Pozzuoli, en las faldas del volcán Vesubio.
} 


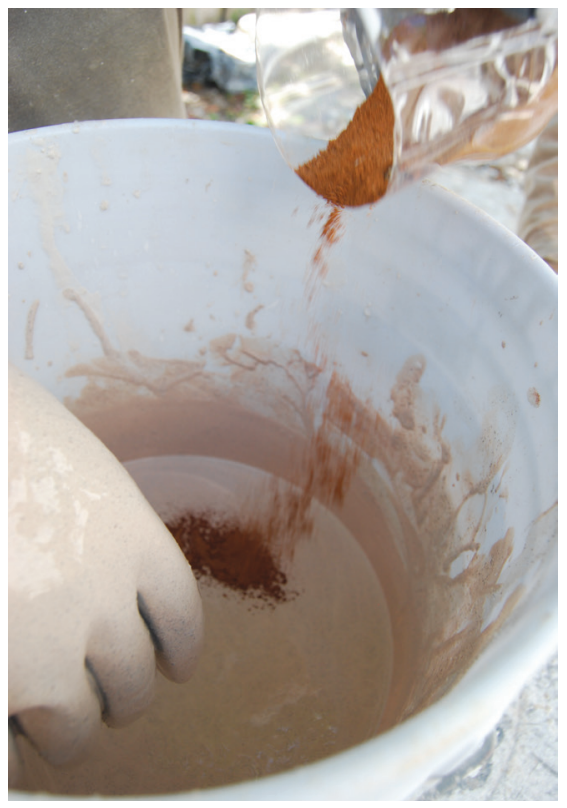

FIGURA 6. Preparación de lechadas puzolánicas de cal, arena fina y polvo de ladrillo (Fotografía: Luis Fernando Guerrero, 2015).

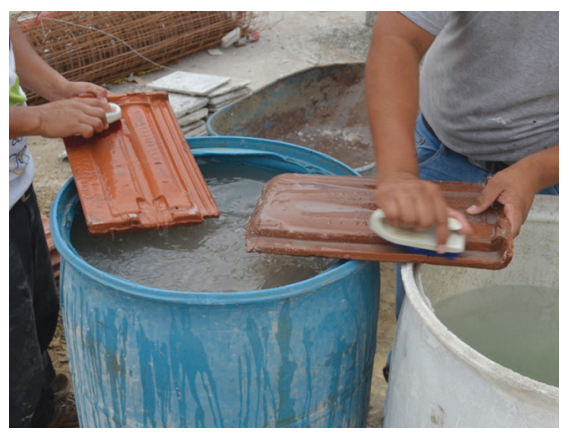

FIGURA 7. Limpieza de las tejas para su reintegración (Fotografía: Geiser Gerardo Martín, 2015).

croorganismos, líquenes y flora parásita albergada tanto sobre los acabados como entre algunos sillares que se encontraban ya descubiertos. De la misma manera, arbustos y árboles invadieron paramentos, remates de muros y pisos, lo que causó desprendimiento de aplanados, fisuras y grietas en muros, así como hundimientos en pisos que llegaron incluso al socavamiento del terreno y a la generación de una grieta en un tramo de la cimentación. Esta vegetación se eliminó cuidadosamente para no disgregar las juntas, mientras que los microorganismos se retiraron, para evitar dañar los elementos de construcción, con cepillos de cerda suave. Gracias a este proceso, se analizaron con más detalle los componentes estructurales del edificio y se identificaron diversas etapas y el cegado de vanos en varias etapas, entre las cuales las más antiguas muestran mayor calidad en el trabajo de la mampostería.

Se detectó el empleo de fragmentos de tejas marsellesas, ladrillos y mosaicos a manera de cuñas, o rajuelas, insertas en las juntas históricas de mortero de cal y arena. Los aplanados históricos evidenciaban, por su parte, la superposición de tres capas de este tipo de morteros en diferentes proporciones y granulometrías, desde los gruesos y semifinos hasta los finos.

En modificaciones que sufrió el edificio, tal vez a fines del siglo XIX, varias secciones específicas de los paramentos se ampliaron con ladrillo, fragmentos de teja, losas de la época y mortero de cal para simular la conformación de pilastras adosadas que, al recibir el aplanado, parecerían ser parte del diseño estructural original, las cuales sólo cumplían, evidentemente, una función decorativa.

Durante la limpieza mencionada se evaluó la pertinencia de eliminar los aplanados incorporados en las intervenciones del 2010 y 2012, hechos a base de cal, arena y cemento, ante lo que se optó por retirarlos por completo. Buena parte de los revoques de cal históricos no requirieron históricas, evidentes en la apertura

más que limpieza, mientras que los faltantes se recubrieron, mediante una técnica constructiva similar a la histórica, con nuevos acabados integrados sobre los paños de muros en los que las juntas ya se habían consolidado y recalzado con rajuelas de teja y ladrillo.

La colocación de rajuela como técnica constructiva hizo posible nivelar fallas leves en las superficies de los muros, lo que tenía el fin de utilizar capas delgadas de aplanados que permitieran su adecuada adherencia, tal y como sucedía con los aplanados originales. A lo largo de este proceso se dejaron expuestas 18 secciones, en las que se localizaron los tubos bajantes de aguas pluviales, de barro vidriado, que, de acuerdo con la propuesta de intervención, se rehabilitarán y pondrán en función nuevamente. Lo mismo sucedió con buena parte de las tejas tipo marsellés que se recuperaron y que se reintegrarán a las cubiertas (Figura 8).

Durante los trabajos descritos con anterioridad se registraron diversas grietas y fisuras, que fue posible consolidar mediante la inyección del material puzolánico antes descrito.

Al finalizar los trabajos de rejunteo y rajueleo de paramentos y columnas, así como de inyección en las partes debilitadas, se procedió a la aplicación inicial, por medio de zarpeo con cuchara de albañil, de agua de cal por aspersión en las zonas

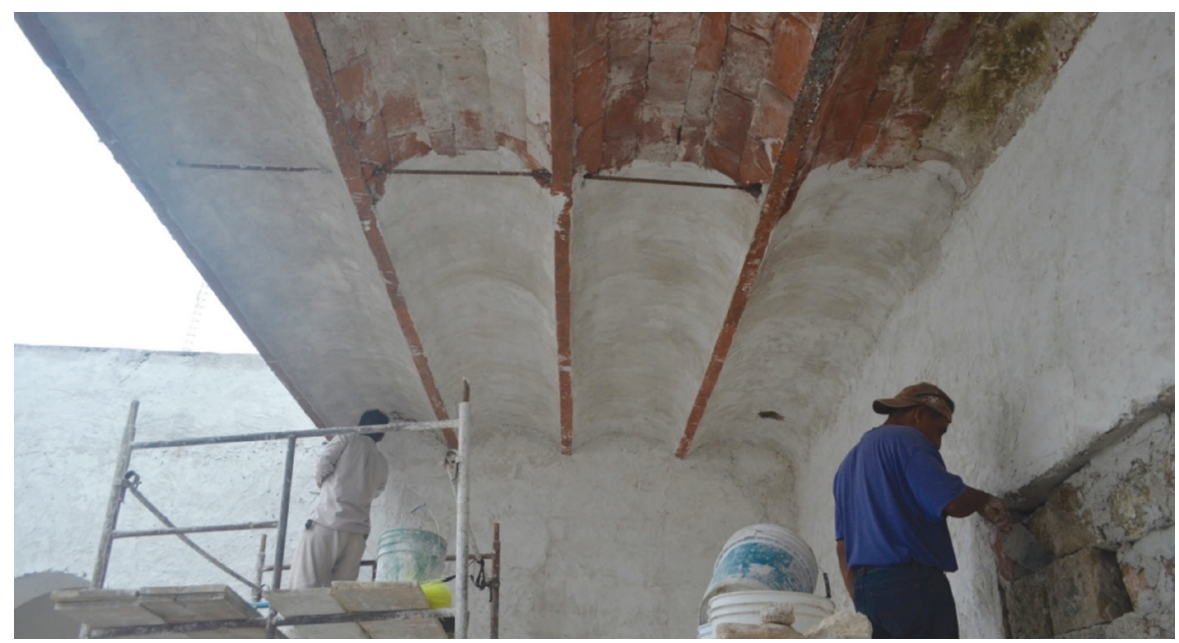

FIGURA 8. Aplicación de aplanados en muros y bajo las bóvedas catalanas (Fotografía: Geiser Gerardo Martín, 2015). 
donde se colocó el primer mortero de cal con arena gruesa. Esto permitió tanto la consolidación de los sillares y juntas originales como la correcta adherencia del nuevo aplanado. Después de esta capa, se realizaron: un cepillado para eliminar excedentes; luego, nuevamente, la aplicación de agua de cal por aspersión, para dar paso a la segunda capa de aplanado, ahora con arena de granulometría media terminada con llana y bruñida con Ilana "de espuma" (flota), para dar un acabado poroso que permitiera la fácil aplicación y adherencia del enlucido final, trabajado con Ilana metálica (Figura 9).

En el patio exterior se procedió a remover pisos de concreto con una cortadora de disco, lo que se realizó por secciones con el fin de no generar percusiones fuertes que produjeran fisuras y grietas en muros y cimentaciones. Al retirar partes de este pavimento, se ubicó el nivel de piso original con restos de piezas de mosaico rojo y negro colocadas en forma de damero y asentadas con mortero de cal. Una sección, conservada in situ, se cubrió con arena y se selló con el nuevo firme, para, así, preservarla en su lugar original. En tanto se trabajaba en la reposición de firmes

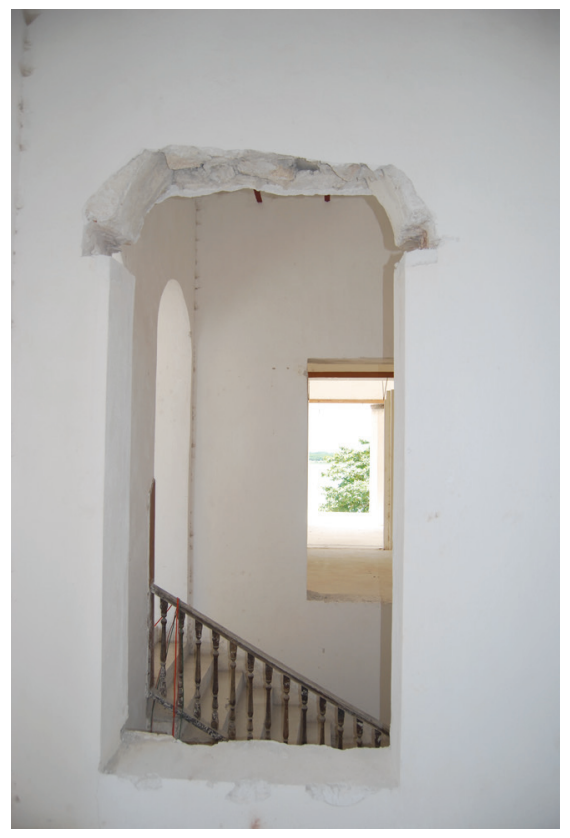

FIGURA 9. Colapso de la platabanda que se apoyaba sobre el dintel de madera (Fotografía: Luis Fernando Guerrero, 2015). y pavimentos del patio, una de las secciones sufrió un hundimiento, por el que se identificó una tubería adosada al muro no registrada en los planos del edificio, la cual parecía seguir la dirección al canal central de desagüe que desemboca en el río. Al dirigir el desnivel, como parte de las nivelaciones de la zona para el nuevo firme, hacia un registro central del desagüe pluvial, se detectaron otras tuberías en diferentes secciones del patio, orientadas, de igual manera, desde los muros hacia el canal. Mediante una revisión más minuciosa de las canalizaciones, se entendió que conformaban un interesante sistema de red pluvial del patio interior que se une a los bajantes pluviales localizados intramuros. Con este hallazgo se procedió a una intervención de registro, rehabilitación y restauración del sistema de manejo pluvial original del inmueble, donde se pudo ver con precisión su tubería de cerámica, la cual presentaba sellos de fabricación con la leyenda: HUGH M. THOMPSON \& CIA. MANUFACTURERS OF SEWER \& DRAIN PIPES. ST. LOUIS. MO. Por este importante dato se logró ubicar cronológicamente la fabricación de estos elementos: entre 1880 y 1908, es decir, en la época en que tuvieron mayor difusión en los Estados Unidos. ${ }^{3}$ Con base en ello es posible estimar, asimismo, la datación aproximada de la elaboración del sistema de desagüe pluvial de la ex aduana.

A la par de estas labores, se llevaron a cabo otras más dentro de los espacios habitables. Tanto en la planta alta como en la baja se analizaron los dinteles de madera de puertas y ventanas, todos seriamente deteriorados por plaga de termita, alojada, incluso, entre las juntas de las dovelas. Hubo que apuntalar todos los arcos en platabanda que constituían los vanos, para así poder retirar los dinteles y fumigar las estructuras du-

\footnotetext{
${ }^{3}$ Esta conclusión se logró con base en inferencias derivadas de información sobre las fechas de fabricación y retiro del mercado de estos elementos constructivos (Anon. s. f.).
}

rante una semana. Posteriormente se integraron los nuevos dinteles, de madera estufada y tratada.

En una ventana de la planta alta que comunicaba una habitación con el cubo de la escalera monumental, el puntal de apoyo de la platabanda se desprendió una vez que se había retirado el dintel de madera, lo que provocó que el relleno se derrumbara hasta la planta baja. Se realizaron calas en los aplanados con el propósito de perfilar las dovelas, y se descubrió que no se trataba de un marco horizontal, como el de la mayoría de los vanos del edificio, sino de un arco de medio punto perfectamente trazado, correspondiente a la primera etapa constructiva del edificio. La supuesta platabanda de descarga del dintel no era, en realidad, sino un "tapón" colocado, probablemente a principios del siglo $\mathrm{XX}$, a manera de tímpano (Figura 10).

Ante esta evidencia se hicieron pequeñas calas sobre los huecos dejados por los dinteles que estaban en proceso de sustitución en otras ventanas y puertas, tanto internas como externas. Pero, al notar que los cuatro primeros vanos sondeados tenían dovelas cortadas con perfiles en platabanda, se puso de manifiesto que sólo el primer caso identificado tenía perfil de medio punto, en tanto que el resto era, efectivamente, de perfil ho-

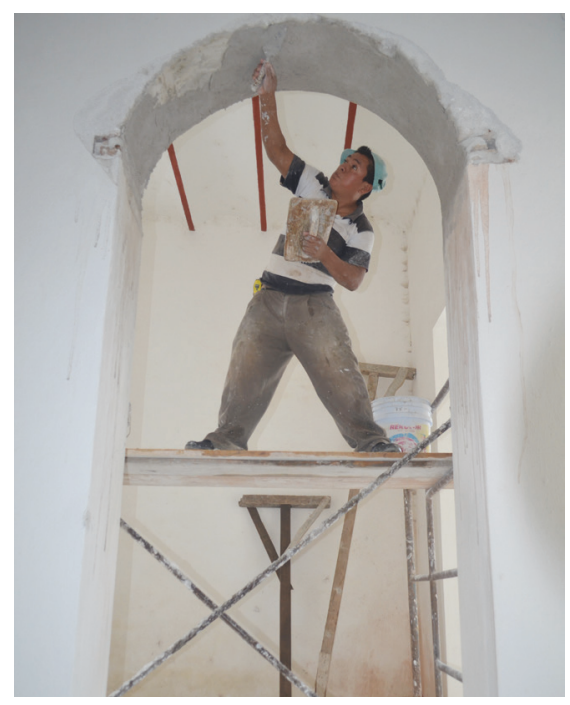

FIGURA 10. Consolidación y aplanado del arco original (Fotografía: Geiser Gerardo Martín, 2015). 
rizontal. En ese caso se liberaron cuidadosamente los restos del tímpano de la segunda historia, con lo que se repusieron y consolidaron los componentes alterados, además de que se colocó un aplanado para la protección del arco original (Figura 11).

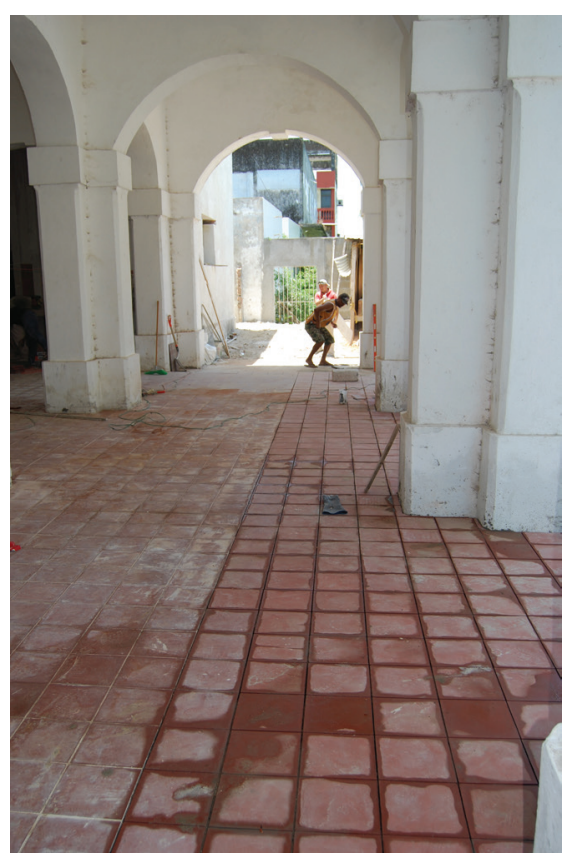

FIGURA 11. Proceso de integración de pisos de loseta de pasta (Fotografía: Luis Fernando Guerrero, 2015).

Otra de las intervenciones de gran impacto para el conjunto fue la integración de pavimentos, que habían tenido diferentes acabados a lo largo de las múltiples adaptaciones del inmueble. En el anterior proyecto de adecuación se había previsto integrarlos con losetas de barro extruido de $20 \times 20 \mathrm{~cm}$, similares a algunos restos históricos localizados en habitaciones de la planta alta. No obstante, en el rediseño de la propuesta se decidió mandar a hacer a Mérida, Yucatán, México, losetas artesanales "de pasta" de color rojo óxido (Figura 11), con base en el supuesto de que el flujo de visitantes a un museo desgastaría en muy poco tiempo los acabados cerámicos.

Se contaba, además, con la evidencia de que a principios del siglo xx la zona de almacenaje de la planta baja contó con un tipo de losetas de pasta parecido al propuesto.
Para instalar los servicios sanitarios requeridos para el correcto funcionamiento del edificio en su nuevo uso como museo se aprovechó un espacio que para el mismo fin se había adaptado a mediados del siglo XX. A pesar de tratarse de una alteración al partido arquitectónico original que se había decidido demoler incluso en el proyecto preliminar, por no considerarse "monumento", su ubicación resultaba muy apropiada para satisfacer las necesidades futuras. Por ello, al local, que se rehabilitó totalmente, se le incorporó un muro húmedo central tanto para dividir los servicios de hombres y mujeres como para concentrar las nuevas instalaciones hidrosanitarias. Esta adaptación se hizo con materiales contemporáneos, y sus acabados incluirán piedra pulida (Figura 12).

Para finalizar, y a la par de los trabajos mencionados hasta el momento, en las plantas alta y baja del inmueble se realizaron las canalizaciones para el cableado eléctrico, distribuidas por piso y colocadas en muros de modo visible, para evitar las perforaciones en componentes patrimoniales, con tubería galvanizada. Como el proyecto museográfico está en proceso, las conexiones eléctricas de cada una de las habitaciones se

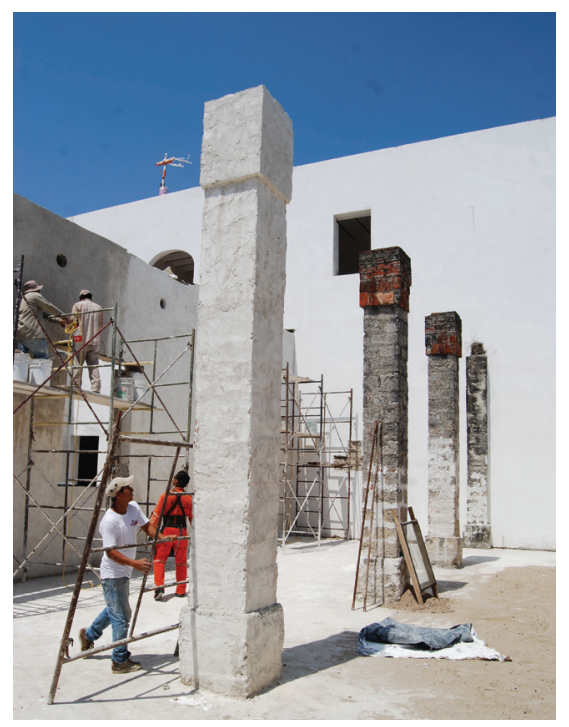

FIGURA 12. Culminación de los revoques de cal y arena antes de la reintegración del techado de armadura y teja de la antigua área de almacenamiento (Fotografía: Luis Fernando Guerrero, 2015). dejaron con cajas a nivel de piso para que exista total flexibilidad en la exposición por venir.

\section{Consideraciones finales}

Ante las necesidades de la vida contemporánea, el patrimonio edificado está constantemente amenazado por la contraposición de intereses políticos, económicos e incluso ideológicos. Aunque existen declaratorias y convenios internacionales, como la Carta de Nairobi (UNESCO 1976) o la de Washington (ICOMOS 1987), que subrayan la importancia de la protección de los conjuntos históricos urbanos y rurales, al buscar su integración a la vida actual suelen pasarse por alto, producto, por un lado, de la inconsciencia e ignorancia de algunos funcionarios e instituciones que aprueban o ejecutan los proyectos de adecuación, como, por el otro, de la pasividad de buena parte de la población, para la que no es prioritaria la conservación del patrimonio edificado (Martín y Reyes 2014:1).

Si este problema es grave para sitios antiguos localizados en centros históricos o zonas arqueológicas reconocidos a escala nacional e internacional, deviene crítico para el caso de poblaciones pequeñas con edificios de escala media, realizados en el siglo XIX, que, además, se encuentran alejados de zonas turísticas; éste es el caso de la ex aduana marítima de Frontera, que, a pesar de que durante casi cien años mantuvo un destacado valor cultural, con una función que impactó fuertemente el desarrollo económico del sureste de la República Mexicana, estuvo a punto de perderse cuando se modificó su función original y, con la construcción de puentes carreteros, decayó la importancia del puerto fluvial.

Este edificio, sin embargo, gracias a la buena factura de su estructura, a su emplazamiento sobre un terreno arenoso consolidado y al uso de materiales como los bloques de conchuela, $y$ los morteros de cal y arena, se conservó en sorprendentes condiciones de estabilidad, sin importar el abandono 
que padeció durante décadas, su mal uso y las intervenciones con materiales constructivos incompatibles.

La puesta en valor de este monumento seguramente incidirá de manera muy favorable en la recuperación de otros inmuebles patrimoniales del puerto de Frontera, así como en la actitud de la población, que los valorará mejor en la medida en que conozca su historia y su vinculación con la ex aduana.

Aunque en fechas recientes se ha insistido en que no es lo más apropiado reutilizar como museos ${ }^{4}$ los inmuebles patrimoniales, pues la comunidad local suele sentirlos como ajenos, para el caso de la región de Centla esta función es clave por la simple razón de que no existen otros.

En este sentido, un diseño museográfico que destaque los valores históricos de la región y explique las cualidades arquitectónicas del edificio, y lo haga considerando la participación comunitaria, será determinante para la sostenibilidad de la intervención (Guerrero 2004). Los costos y el impacto ambiental que implican las obras de restauración hacen impostergable buscar los mecanismos de integración y puesta en valor de los edificios y sus espacios para que formen parte de la nueva dinámica urbanística del siglo XXI (Martín y Reyes 2014:1; Ballart 2006:34).

Al trascender en el tiempo y permanecer activo, el patrimonio edificado sigue vigente en la memoria de los pobladores, con lo cual refuerza $-\mathrm{y}$, en algunos casos, forja- identidades y sentido de pertenencia (Martín 2013:174).

Como se ha repetido a lo largo del siglo XX en casi todos los documentos normativos de la conservación, sin la utilización social del patrimonio, éste pierde toda oportunidad de

\footnotetext{
${ }^{4}$ Un ejemplo en este sentido es el Museo de Historia de Tabasco, Casa de los Azulejos, que, según nuestra apreciación, a pesar de que se localiza en una de las zonas más vitales de Villahermosa: en pleno centro histórico (IEC-GT 2015), cuenta con bajísima afluencia de visitantes locales.
}

preservación. Si la recuperación de espacios del pasado sólo apuesta por la llegada de turistas, éstos acabarán por dañar progresivamente los vestigios históricos y por trastocar no sólo la economía local sino, incluso, la propia identidad cultural. En nuestros días se ha vuelto urgente dar cara al reto de conservar el patrimonio a partir del principio de la identificación, valoración y apropiación por parte de las comunidades locales (Guevara y Valdespín 2010).

Para ello es indispensable buscar los mecanismos que permitan ampliar la perspectiva acerca de lo que se considera patrimonio histórico-cultural en México, con el fin de borrar gradualmente la inoperante separación que la Ley Federal de Monumentos Arqueológicos, Artísticos e Históricos (CEUM 1972) ha impuesto a los monumentos, jerarquizados por su antigüedad, al considerar, como reza su artículo 46, que "Para los efectos de competencia, el carácter arqueológico de un bien tiene prioridad sobre el carácter histórico, y éste a su vez sobre el carácter artístico" (Guerrero 2004). Es momento de volver a plantearse la reutilización de todo el patrimonio, sea de la época que sea, como medio para elevar la calidad de vida de la sociedad que lo rodea, calidad entendida no desde el punto de vista economicista del término, sino a partir de una visión que pondere el tejido de las relaciones comunitarias y la interacción con el entorno natural.

\section{Referencias}

Anon

s. f. Lanchas en el muelle. Fotos antiguas de Frontera, Tabasco, documento electrónico disponible en [http://www.mexicoenfotos.com/antiguas/tabasco/frontera/ MX14089123670056], consultado en marzo del 2015.

s. f. The History of Sanitary Sewers [página web], documento disponible en [http://www.sewerhistory.org/ photosgraphics/pipes-clay/], consultado el 7 de diciembre del 2015.
Ballart, Josep

2006 El patrimonio histórico y arqueológico: valor y uso, Barcelona, Ariel.

Barrera Bassols, Marco

1989 "La investigación histórica para el museo de la navegación de Frontera, Tabasco", tesis para obtener el grado de licenciatura en historia, México, ENAH-INAH.

Chávez, Ulises

2007 "Potonchán y Santa María de la Victoria: una propuesta geomorfológico/arqueológica a un problema histórico", Estudios de Cultura Maya, XXIX:103-139.

CEUM

1972 "Ley Federal de Monumentos y Zonas Arqueológicos, Artisticos e Históricos", Diario Oficial de la Federación, Congreso de los Estados Unidos Mexicanos (CEUM), 6 de mayo (última reforma publicada: 13 de enero del 1986).

Díaz del Castillo, Bernal 2007 [1632] Historia verdadera de la conquista de la Nueva España, México, Porrúa.

Digaohm-Semar

s. f. Frontera, Tabasco, Dirección General Adjunta de Oceanografía, Hidrografía y Meteorología, Digaohm-Semar, SECRETARÍA DE MARINA documento electrónico disponible en [http://digaohm.semar.gob.mx/cuestionarios/cnarioFrontera.pdf], consultado en marzo del 2015.

García, Olinda 1996 Historia y geografía del estado de Tabasco, México, Santillana.

Guerrero, Luis

2004 "El valor tipológico del patrimonio edificado", Anuario Investigación y Diseño 2004, México, UAM-X, 83-96.

2014 "Arquitectura vernácula y conservación sostenible", La Gaceta del Instituto del Patrimonio Cultural, 27:26-32.

Guevara, Miguel y Michelle Valdespín 2010 "Arqueología y proyectos comunitarios. Objetivos y estrategias", en Memorias del 1. ${ }^{\text {er }}$ Congreso de Patrimonio Cultural en México: Problemática Actual y Propuestas para su Intervención, Mérida, Asociación 
Yucateca de Especialistas en Restauración y Conservación del Patrimonio Edificado, A. C./UAdY.

ICOMOS

1987 "Carta Internacional para la Conservación de Ciudades Históricas y Áreas Urbanas Históricas", Carta de Washington, Asamblea General del Consejo Internacional de Sitios y Monumentos (ICOMOS), Washington, D. C.

IEC-GT

2015 Museo de Historia de Tabasco, Casa de los Azulejos [página web], Instituto Estatal de Cultura, Gobierno de Tabasco (IEC-GT), documento electrónico disponible en [http:// iec.tabasco.gob.mx/content/museohistoria-tabasco], consultado en diciembre del 2015.
Martín, Geiser

2013 "Entre montes y solares: el valor y uso local de construcciones mayas en comunidades del sur del estado de Yucatán", en Juan García Targa (ed.), Patrimonio cultural mexicano: modelos explicativos, Archaeopress/British Archaeological Reports, International Series, 173-182.

Martín, Geiser y A. Reyes

En prensa "Gestión y conservación del patrimonio cultural edificado. Una aproximación a la valorización del Centro Histórico de la ciudad de Mérida", en Juan García Targa (ed.), Cultura y patrimonio mexicano del siglo XXI, Archaeopress/British Archaeological Reports, International Series, 2014.
UNESCO

1976 "Acta de la conferencia General 19. a reunión Nairobi", Resolución 0.1 aprobada por la Conferencia General en su $19 .^{\text {a }}$ reunión, 26 de octubre al 30 de noviembre.

México en fotos

2015 Fotografías de la ex aduana de Tabasco [página web], documento electrónico disponible en [www. mexicoenfotos.com], consultado en diciembre del 2015.

Ortiz, Neftalí

2015 "Casi listo el Museo de la Navegación", Tabasco [página web], documento electrónico disponible en [http://www.tabascohoy.com/2/ notas/270579/casi-listo-el-museode-navegacion], consultado en diciembre del 2015. 


\section{Geiser Gerardo Martín Medina}

Universidad Autónoma de Yucatán (UADY), México

arqlgo_geisermartinmedina@hotmail.com

Licenciado en arqueología (Universidad Autónoma de Yucatán [UADY], México), institución en la que participó en proyectos como el de Arqueología Histórica en la Hacienda San Pedro Cholul, habiendo presentado varias conferencias y publicaciones. Ha colaborado, además, en dos salvamentos y el registro de deterioros en los sitios arqueológicos de Sayil, Labná, X’lapak y Kabah, en Yucatán, México, así como en el Salvamento Arqueológico Comalcalco y el Proyecto Arqueológico Comalcalco, conservación integral de los edificios de la Gran Acrópolis, en Tabasco, México. Es miembro de la Society for American Archaeology (SAA, Sociedad para la Arqueología Americana), de la Society for Historical Archaeology (SHA, Sociedad para la Arqueología Histórica) y de Xíimbal K'áax, Conservación, Investigación y Difusión del Patrimonio Cultural y Natural A. C., en Mérida, Yucatán. Ha presentado 34 trabajos en congresos nacionales e internacionales y actualmente asesora a la empresa que restaura la ex aduana marítima de Frontera, Tabasco, México.

\section{Luis Fernando Guerrero Baca}

Universidad Autónoma Metropolitana (UAM), México luisfg1960@yahoo.es

Licenciado en arquitectura (Universidad Autónoma Metropolitana [UAM], México); maestro en restauración arquitectónica (Escuela Nacional de Conservación, Restauración y Museografía, Instituto Nacional de Antropología e Historia [ENCRyM, INAH], México), y doctor en diseño con especialidad en conservación del patrimonio (UAM, México). Realiza investigaciones sobre tipología y teoría de la conservación, así como materiales y sistemas constructivos tradicionales, habiendo escrito más de 70 artículos en publicaciones mexicanas y extranjeras, e impartido más de 150 conferencias y talleres nacionales e internacionales. Es profesor-investigador de la UAM, México, desde 1987 y profesor invitado en los posgrados en conservación de la ENCRYM-INAH, la Universidad Nacional Autónoma de MéxiCo (UNAM), de la Universidad Autónoma del Estado de México (UAEM), la Universidad Michoacana de San Nicolás de Hidalgo (UMSNH), la Universidad Autónoma de Tamaulipas (UAT), todas las anteriores en México; la Universidad de Cuenca (UCuenca), en Ecuador, y la Universidad Mayor de San Andrés (UMSA), en Bolivia. Es miembro del Sistema Nacional de Investigadores (SNI), la Red Iberoamericana PROTERRA y la Chaire UNESCO (cátedra UNESCO): Architectures de Terre, Cultures Constructives et Développement Durable; consultor del World Heritage Committee (WHC, Comité de Patrimonio Mundial, UNESCO), y miembro experto del International Scientific Committee on Earthen Architectural Heritage, International Council on Monuments and Sites (ISCEAH-ICOMOS, Comité Científico Internacional de Patrimonio Arquitectónico de Tierra, Consejo Internacional de Monumentos y Sitios).

Postulado/Submitted: 04.05.15

Aceptado/Accepted: 23.12 .15

Publicado/published: 31.03 .2016 